\title{
Clinical diagnosis and mutation analysis of four Chinese families with succinic semialdehyde dehydrogenase deficiency
}

Ping Wang ${ }^{1 \dagger}$, Fengying $\mathrm{Cai}^{2+}$, Lirong $\mathrm{CaO}^{3}$, Yizheng Wang $^{3}$, Qianqian Zou ${ }^{3}$, Peng Zhao ${ }^{4}$, Chao Wang ${ }^{1}$, Yuqin Zhang ${ }^{5}$, Chunquan Cai ${ }^{6^{*}}$ and Jianbo Shu ${ }^{1^{*}}$ (i)

\begin{abstract}
Background: Succinic semialdehyde dehydrogenase (SSADH) deficiency is a rare autosomal recessively-inherited defect of $\gamma$-aminobutyric acid (GABA) metabolism. The absence of SSADH, which is encoded by aldehyde dehydrogenase family 5 member A1 (ALDH5A1) gene, leads to the accumulation of GABA and $\gamma$-hydroxybutyric acid (GHB). Few cases with SSADH deficiency were reported in China.

Case presentation: In this study, four Chinese patients were diagnosed with SSADH deficiency in Tianjin Children's Hospital. We conducted a multidimensional analysis with magnetic resonance imaging (MRI) of the head, semi quantitative detection of urine organic acid using gas chromatography-mass spectrometry, and analysis of ALDH5A1 gene mutations.

Two of the patients were admitted to the hospital due to convulsions, and all patients were associated with developmental delay. Cerebral MRI showed symmetrical hyperintense signal of bilateral globus pallidus and basal ganglia in patient 1; hyperintensity of bilateral frontal-parietal lobe, widened ventricle and sulci in patient 2; and widened ventricle and sulci in patient 4. Electroencephalogram (EEG) revealed the background activity of epilepsy in patient 1 and the disappearance of sleep spindle in patient 2. Urine organic acid analysis revealed elevated GHB in all the patients. Mutational analysis, which was performed by sequencing the 10 exons and flanking the intronic regions of ALDH5A1 gene for all the patients, revealed mutations at five sites. Two cases had homozygous mutations with c. $1529 \mathrm{C}>\mathrm{T}$ and $\mathrm{c} .800 \mathrm{~T}>\mathrm{G}$ respectively, whereas the remaining two had different compound heterozygous mutations including c.527G > A/c.691G > A and c.1344-2delA/c.1529C > T. Although these four mutations have been described previously, the homozygous mutation of c.800 T $>G$ in ALDH5A1 gene is a novel discovery.
\end{abstract}

Conclusion: SSADH deficiency is diagnosed based on the elevated GHB and 4, 5DHHA by urinary organic acid analysis. We describe a novel mutation p.V267G (c.800 T > G) located in the NAD binding domain, which is possibly crucial for this disease's severity. Our study expands the mutation spectrum of ALDH5A1 and highlights the importance of molecular genetic evaluation in patients with SSADH deficiency.

Keywords: Succinic semialdehyde dehydrogenase deficiency, ALDH5A1 gene, Multidimensional analysis, Novel mutation

\footnotetext{
*Correspondence: tjpns@126.com; shjb1981@sina.com

†Ping Wang and Fengying Cai contributed equally to this work.

${ }^{6}$ Department of Neurosurgery, Tianjin Children's Hospital, No.238, Longyan

Road, Beichen District, Tianjin 300134, People's Republic of China

${ }^{1}$ Tianjin Pediatric Research Institute, Tianjin Children's Hospital, No.238,

Longyan Road, Beichen District, Tianjin 300134, People's Republic of China

Full list of author information is available at the end of the article
}

(c) The Author(s). 2019 Open Access This article is distributed under the terms of the Creative Commons Attribution 4.0 International License (http://creativecommons.org/licenses/by/4.0/), which permits unrestricted use, distribution, and reproduction in any medium, provided you give appropriate credit to the original author(s) and the source, provide a link to the Creative Commons license, and indicate if changes were made. The Creative Commons Public Domain Dedication waiver (http://creativecommons.org/publicdomain/zero/1.0/) applies to the data made available in this article, unless otherwise stated. 


\section{Background}

Succinic semialdehyde dehydrogenase (SSADH, OMIM: 271980) deficiency, also known as 4-hydroxybutyric aciduria, is a rare autosomal recessive genetic disorder that affects the degradation of $\gamma$-aminobutyric acid [1]. SSADH catalyzes succinic semialdehyde (SSA) to succinic acid (SA) in the final step of $\gamma$-aminobutyric acid (GABA) catabolism. In the absence of SSADH, SSA is converted into $\gamma$-hydroxybutyric acid (GHB) and other related metabolites. GHB can be converted to 3-deoxytetronic acid (3DT) or 2-deoxytetronic acid (2DT) via $\alpha$ or $\beta$ oxidation. GABA and GHB accumulate in the brain, blood, urine, and cerebral spinal fluid. The clinical features of this disease are various and non-specific, including developmental delay, intellectual deficit, hypotonia, ataxia and seizures, and especially language delays [2-4]. Severity ranges from mild impairment to a relentlessly progressive neurodegenerative course with intractable seizures in infancy [5]. $\mathrm{GABA}_{\mathrm{A}}$ receptor down regulation was previously reported as one of the pathophysiological mechanisms [2], but current data reveal a surprisingly complex pattern of pathophysiology including oxidative stress parameters and dysregulation of autophagy and mitophagy [1].

The gene of $A L D H 5 A 1$ with a full length of $1605 \mathrm{bp}$ containing 10 exons, located in chromosome 6p22.3, and codes 535 amino acids with the first 47 residues representing the mitochondrial targeting peptide $[4,6]$. The monomeric SSADH consisted of three domains: an oligomerization domain (174-195 and 525-535), a catalytic domain (308-508), and an N-terminal NAD-binding domain (residues 48-173, 196-307, and 509-524) that is responsible for the binding of $\mathrm{NAD}^{+}$to the enzyme [7]. To date, more than 56 mutations of $A L D H 5 A 1$ have been identified [8] and are considered as the main cause for the decrease or elimination of SSADH activity.

Only a few cases of SSADH deficiency have been reported in mainland China [9-12]. In this study, we summarized the characteristics of clinical data and analyzed $A L D H 5 A 1$ gene mutations in four Chinese patients with SSADH deficiency diagnosed in our hospital. We present a novel mutation (c.800 $\mathrm{T}>\mathrm{G}$ ) in ALDH5A1, which enriches the analysis of $A L D H 5 A 1$ mutation spectrum. Based on our correlation analysis between SSADH protein function and the clinical data of patients, we speculate that the mutation in NAD binding domain (p.S510F and p.V267G) is crucial for this disease's severity.

\section{Case presentation}

Four Chinese patients (two males and two females) at the age of 86 days to 5 years were diagnosed with SSADH deficiency in Tianjin Children's Hospital in China from June 2012 to September 2016. These patients had no family history. Detailed data are shown in Table 1. Cerebral magnetic resonance imaging (MRI), electroencephalogram (EEG), and routine tests were conducted. Semi-quantitative analysis of organic acids in urine was performed by gas chromatography-mass spectrometry (GC/MS), and the results were included in Table 2. Genetic analysis was performed using blood samples collected from the patients and their parents. One hundred healthy children were enrolled as the control group. DNA was extracted by gDNA Mini Kit (BIOMIGA, USA) according to the manufacturer's instructions. PCR amplification for 10 exons and flanking intronic regions of ALDH5A1 gene was performed, whose primers and reaction condition were designed based on relevant references [12]. PCR products were electrophoresed on $2 \%$ agarose gels and sequenced by biological company (GENEWIZ, Tianjin). The sequencing results were compared with the reference sequence of ALDH5A1 (NM_170740) of NCBI.

The alignment for amino acid sequence of SSADH in Human, Chimpanzee, Bos, Rattus, Anser, Xenopus, and Zebrafish were conducted by DNAMAN software. The functional prediction of gene mutation was performed by Polyphen2 (http://genetics.bwh.harvard.edu/pph2/) and SIFT (http://sift.jcvi.org/).

\section{Patient 1}

Patient 1 was a 3 -year-old female born at term by cesarean delivery with the history of aspiration of amniotic fluid and was not awaked easily for one day after birth. She was admitted to the hospital due to cough for 5 days and intermittent convulsions for 3 days. She had partial convulsions without fever while awake, she slept after remission, most

Table 1 Clinical data of 4 Chinese patients affected with SSADH deficiency

\begin{tabular}{|c|c|c|c|c|}
\hline Patients & 1 & 2 & 3 & 4 \\
\hline Gender & $\mathrm{F}$ & M & M & $F$ \\
\hline Age of diagnosis & $3 y$ & $86 d$ & $5 y$ & $8 m$ \\
\hline Cesarean delivery & Y & Y & $\mathrm{N}$ & $\mathrm{N}$ \\
\hline Family history & $\mathrm{N}$ & $\mathrm{N}$ & $\mathrm{N}$ & $N$ \\
\hline Consanguineous & $\mathrm{N}$ & $\mathrm{N}$ & Y & $\mathrm{N}$ \\
\hline Convulsions & Y & Y & $\mathrm{N}$ & $\mathrm{N}$ \\
\hline Developmental delay & Y & Y & Y & Y \\
\hline Hypotonia & $\mathrm{N}$ & $\mathrm{N}$ & $\mathrm{N}$ & Y \\
\hline Hyporeflexia & $\mathrm{N}$ & $\mathrm{N}$ & $\mathrm{N}$ & Y \\
\hline Sleep disturbances & $\mathrm{N}$ & Y & $\mathrm{N}$ & Y \\
\hline Abnormal EEG & Y & Y & UN & $N$ \\
\hline Abnormal MRI & Y & Y & UN & Y \\
\hline Metabolic acidosis & $\mathrm{N}$ & Y & $N$ & $N$ \\
\hline 4-HB urine & Y & Y & Y & Y \\
\hline
\end{tabular}

$F$ female, $M$ male, $y$ years, $d$ days, $m$ months, $N$ no, $Y$ yes, $U N$ unknown 
Table 2 Analysis of organic acid in urine by GC/MS

\begin{tabular}{llllll}
\hline Metabolites & Case1 & Case2 & Case 3 & Case 4 & Reference range \\
\hline 4-hydroxybutyric acid & 1.533 & 4.887 & 2.178 & 9.603 & $<0.001$ \\
3,4-dihydroxybutanoic acid & 1.695 & 9.073 & 1.796 & 16.173 & $<0.895$ \\
2,4-dihydroxybutanoic acid & 0.549 & 1.425 & 0.788 & 8.216 & $<0.208$ \\
4,5-dihydroxyhexanoic acid & 0.958 & 6.320 & 1.548 & 4.197 & $<0.001$ \\
\hline
\end{tabular}

Value: metabolite peak area ratio to creatinine

convulsions were less than two minutes in duration. She had no movement or language disorders during the postictal period. She also presented with developmental delay, especially language delay. Cerebral MRI displayed symmetrical hyperintensity of bilateral basal ganglia and globus pallidus on T2-weighted image (T2WI) and hyperintensity of the bilateral parietal lobe white matter on fluid-attenuated inversion recovery (FLAIR) imaging. EEG revealed high voltage of delta and theta wave at approximately $2-4 \mathrm{~Hz}$. Urinary organic acid analysis showed elevated GHB concentrations.

Molecular analysis of ALDH5A1 confirmed a compound heterozygous mutation of c.527G > A/c.691G > A (p.G176E/p.E231K) in exons 3 and 4. c.527G > A was inherited from her mother, whereas c.691G $>$ A was inherited from her father. We have previously reported these two mutations $[8,12]$. Bioinformatics analysis indicated that p.G176 and p.E231 are highly conserved among species. These mutations were predicted to be "Probably damaging" and "Possibly damaging" based on the Polyphen2 software.

\section{Patient 2}

Patient 2 is an 86-day-old male conceived through in vitro fertilization and born at 37 weeks by cesarean section due to cord around the neck. His mother had an abnormal reproductive history. Her first baby died of convulsion at 23 days after birth, while her second pregnancy was ectopic. This patient was referred to the hospital due to intermittent convulsions for more than 2 months and exacerbation for 2 days. Partial convulsions occurred while he was awake or asleep without fever. He was back to normal without movement disorders. He presented with decreased attention, poor head control, and thumb abduction. Cerebral MRI revealed patchy hyperintensity in bilateral frontal and parietal lobe on T2WI. Widened ventricle and the interval outside the cerebral were also noted in the cerebral MRI. EEG showed sleep spindle asynchrony. Blood gas analysis indicated metabolic acidosis. GHB in urine was significantly elevated according to urinary organic acid analysis. Unfortunately, this patient died of pneumonia despite rescue efforts.

A homozygous mutation variant, c.1529C > T (p.S510F), was identified in exon 10 of the ALDH5A1 gene in this patient. Subsequent targeted mutational analysis of exon 10 of his mother confirmed the segregation of the variant. The healthy father did not carry the sequence variant. p.S510 is highly conserved, its mutation is predicted to be "Probably damaging."

\section{Patient 3}

Patient 3 was a 5 -year-old boy born at full term with the history of hypoxia after birth. He was admitted to the hospital due to developmental delay complicated with lack of sleep. Urinary organic acid analysis showed a high level of GHB. EEG and cerebral MRI were unclear because both were performed by other hospitals.

This patient harbored a novel homozygous mutation of c.800 T > G (p.V267G) in exon 5, which he inherited from his parents. Bioinformatics analysis revealed that p.V267 is highly conserved among species. The mutation
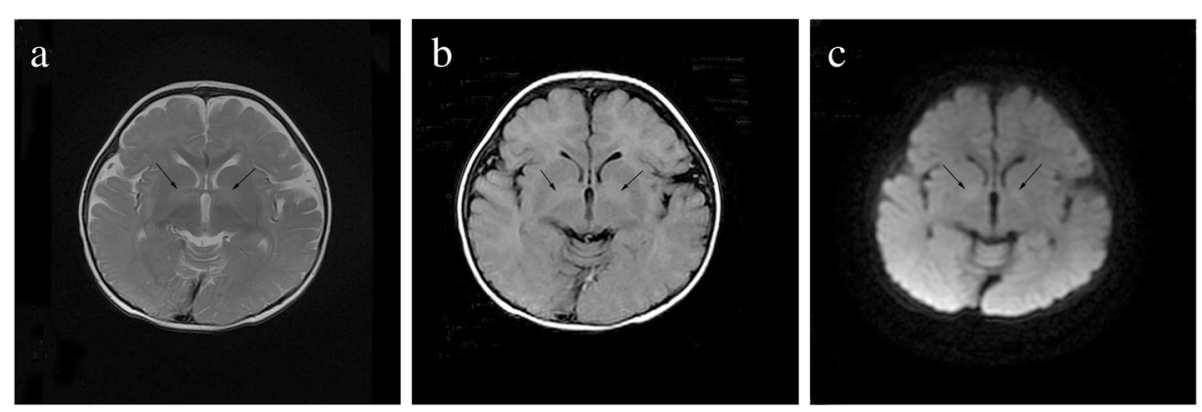

Fig. 1 Cerebral MRI of patient 4 displayed hyperintense signal of bilateral globuspallidus (arrow), widened ventricle, and interval outside the cerebral on T2WI (a), FLAIR (b) and diffusion weighted image (c) 
was predicted to be "probably damaging" and "disease causing" with a score of approximately equal to 1 .

\section{Patient 4}

Patient 4 was an 8-month-old girl born at full term by natural delivery and referred to the hospital because of developmental delay. The clinical manifestations showed slow pupillary light reflex and hyporeflexia and hypotonia of all limbs. Cerebral MRI revealed widened ventricle, cistern, and sulci, which was considered as delayed myelination (Fig.1). EEG was normal. Urinary organic acid analysis showed an increased excretion of GHB.

A compound heterozygous mutation of c.1344-2delA/ c.1529C > T was identified in exons 9 and 10 in this girl. The mutation c.1344-2delA located in the intron splice site, and p.S510F $(\mathrm{c} .1529 \mathrm{C}>\mathrm{T})$ belongs to the NAD-binding domain. These mutations are separately inherited from both of her parents.

\section{Discussion and conclusion}

SSADH deficiency is a rare inherited metabolic disease characterized by the metabolic disorder of GABA. The incidence rate is unknown but has been estimated at 1:100000 [1]. The first case was reported by Jakobs in 1981 [13]. Parental consanguinity has been reported in approximately $40 \%$ of affected population [9]. The median age at diagnosis of SSADH is 2 years old, but underdiagnosis is suspected [14]. Nearly $80 \%$ of patients are diagnosed at the age of 5 years, although a few ones are confirmed in late childhood or adolescence [14], and sporadic patients are diagnosed during adulthood [15]. In this study, patient 3 was born to consanguineous parents. These four Chinese patients were diagnosed at the age range of 86 days to 5 years. The signs and symptoms of SSADH deficiency are often non-specific, including developmental delay, hypotonia, hyporeflexia, ataxia, neuropsychiatric problems, and epilepsy. Hypotonia and neuropsychiatric problems each have occurred in $70 \%$ of patients, and approximately half of these patients have epilepsy [16]. Neuropsychiatric symptoms, involving disabling obsessive compulsive disorder and anxiety in addition to inattention, hyperactive behavior, and sleep disturbances, were also noted [17]. EEG shows generalized spike-waves, photosensitivity discharge, and sleep spindle asynchrony [18]. In this study, patients 1 and 2 presented with convulsions, and all the patients had the history of developmental delay. Patient 4 displayed hypotonia and hyporeflexia. Patient 2 and 4 exhibited decreased attention and sleep disturbances, respectively. EEG showed the background of epilepsy in patient 1 and the disappearance of sleep spindle in patient 2 . The clinical manifestations of these patients are consistent with the signs and symptoms of SSADH deficiency.
In this study, we identified four patients with different ALDH5A1 gene mutations. The c.800 T > G (p.V267G) mutation in patient 3 is a novel mutation. Bioinformatics analysis revealed that this mutation was highly conserved across species and might play an important biological role. This mutation was predicted to be "probably damaging" and "disease causing" based on the Polyphen2 software with a score of approximately equal to $1.267 \mathrm{~V}$ is located in the N-terminal NAD-binding domain, the mutation in this domain may influence the combination of SSADH with the $\mathrm{NAD}^{+}$molecule.

SSADH deficiency is diagnosed based on the elevated GHB and 4, 5DHHA by urinary organic acid analysis. SSADH activity or molecular genetic analysis of ALDH5A1 gene are used to confirm this disorder [4]. In this study, the GHB in urine was elevated dramatically in these four Chinese patients. Genetic investigation also identified five mutations of $A L D H 5 A 1$ gene. Cerebral MRI showed symmetrical hyperintense signal of bilateral globus pallidus and basal ganglia in patient 1 ; hyperintensity of bilateral frontal-parietal lobe, widened ventricle, and sulci in patient 2; and widened ventricle and sulci in patient 4 .

In conclusion, we reported five $A L D H 5 A 1$ gene mutations in four Chinese children with SSADH deficiency. Among these mutations, one novel mutation is presented uniquely in our study. Our study may provide useful information for the gene mutation analysis of ALDH5A1 and contribute to the diagnosis of SSADH deficiency in clinical practices and in the screening of $A L D H 5 A 1$ gene mutation carriers, especially those undergoing neonatal screening with family histories.

\section{Abbreviations}

4,5DHHA: 4,5-dihydroxyhexanoic acid; ALDH5A1: Aldehyde dehydrogenase family 5 member A1; FLAIR imaging: Fluid-attenuated inversion recovery imaging; GABA: Gamma-aminobutyric acid; GC/MS: Gas chromatographymass spectrometry; GHB: -hydroxybutyric acid; MRI: Magnetic resonance imaging; SNPs: Single nucleotide polymorphisms; SSADH: Succinic semialdehyde dehydrogenase

\section{Acknowledgments \\ The authors would like to thank all the study participants.}

\section{Funding}

This research is supported by Natural Science Foundation of Tianjin City (16JCQNJC11900), Key Project of Tianjin Health Care Professionals (16KG166), National Natural Science Foundation of China (81771589), and The Program of Tianjin Science and Technology Plan (18ZXDBSY00170). None of the funding bodies played any role in the design of the study or the collection, analysis, or interpretation of data, nor in writing of the manuscript.

Availability of data and materials

Datasets are available from the corresponding author on reasonable request.

Authors' contributions

IS contributed to conception and design of the study. PW was involved in the design of this study, genetic analysis of the ALDH5A1 gene and writing the manuscript. FC performed the analysis and interpretation of data. LC, YW, QZ and CW participated in bioinformatics analysis and interpretation of the results. PZ and YZ provided clinical diagnosis and genetic counseling. CC 
participated in supervision of the project, obtained funding and revising the final manuscript. All authors read and approved the final manuscript.

\section{Ethics approval and consent to participate}

Written Informed consents were obtained from guardians and the approval of the Medical Ethics Committee of Tianjin Children's Hospital.

\section{Consent for publication}

Written informed consents were obtained from the guardians to participate in this study and for the publication of the data.

\section{Competing interests}

The authors declare that they have no competing interests.

\section{Publisher's Note}

Springer Nature remains neutral with regard to jurisdictional claims in published maps and institutional affiliations.

\section{Author details}

'Tianjin Pediatric Research Institute, Tianjin Children's Hospital, No.238, Longyan Road, Beichen District, Tianjin 300134, People's Republic of China. ${ }^{2}$ Department of Physiology, Tianjin Medical College, Tianjin 300222, China. ${ }^{3}$ Graduate College of Tianjin Medical University, Tianjin 300070, China. ${ }^{4}$ Department of Rehabilitation, Tianjin Children's Hospital, Tianjin 300134, China. ${ }^{5}$ Department of Neurology, Tianjin Children's Hospital, Tianjin 300134, China. ${ }^{6}$ Department of Neurosurgery, Tianjin Children's Hospital, No.238, Longyan Road, Beichen District, Tianjin 300134, People's Republic of China.

Received: 9 December 2018 Accepted: 7 May 2019

Published online: 22 May 2019

\section{References}

1. Malaspina P, Roullet JB, Pearl PL, Ainslie GR, Vogel KR, Gibson KM. Succinic semialdehyde dehydrogenase deficiency (SSADHD): pathophysiological complexity and multifactorial trait associations in a rare monogenic disorder of GABA metabolism. Neurochem Int. 2016;99:72-84.

2. Horino A, Kawawaki H, Fukuoka M, Tsuji H, Hattori Y, Inoue T, et al. A case of succinic semialdehyde dehydrogenase deficiency with status epilepticus and rapid regression. Brain and Development. 2016;38:866-70.

3. Akiyama T, Osaka H, Shimbo H, Kuhara T, Shibata T, Kobayashi K, et al. SSADH deficiency possibly associated with enzyme activity-reducing SNPS. Brain and Development. 2016:38:871-4.

4. Tay CG, Ariffin H, Yap S, Rahmat K, Sthaneshwar P, Ong LC. Succinic Semialdehyde dehydrogenase deficiency in a Chinese boy: a novel ALDH5A1 mutation with severe phenotype. J Child Neurol. 2015;30:927-31.

5. Lapalme-Remis S, Lewis EC, De Meulemeester C, Chakraborty P, Gibson KM, Torres $C$, et al. Natural history of succinic semialdehyde dehydrogenase deficiency through adulthood. Neurology. 2015:85:861-5.

6. Leo S, Capo C, Ciminelli BM, lacovelli F, Menduti G, Funghini S, et al. SSADH deficiency in an Italian family: a novel ALDH5A1 gene mutation affecting the succinic semialdehyde substrate binding site. Metab Brain Dis. 2017:32:1383-8

7. Kim YG, Lee S, Kwon OS, Park SY, Lee SJ, Park BJ, et al. Redox-switch modulation of human SSADH by dynamic catalytic loop. EMBO J. 2009;28:959-68.

8. Shu J, Cai F, Fan W, Meng Y, Zhang C, Cai C, et al. Identification of ALDH5A1 gene mutations in a Chinese family affected with succinic semialdehyde dehydrogenase deficiency. Zhonghua Yi Xue Yi Chuan Xue Za Zhi. 2017:34:6-9.

9. Li X, Ding Y, Liu Y, Zhang Y, Song J, Wang Q, et al. Succinic semialdehyde dehydrogenase deficiency of four Chinese patients and prenatal diagnosis for three fetuses. Gene. 2015;574:41-7.

10. Ma YY, Wu TF, Liu YP, Wang Q, Li XY, Ding Y, et al. Two compound frameshift mutations in succinate dehydrogenase gene of a Chinese boy with encephalopathy. Brain and Development. 2014;36:394-8.

11. Liu N, Kong XD, Kan QC, Shi HR, Wu QH, Zhuo ZH, et al. Mutation analysis and prenatal diagnosis in a Chinese family with succinic semialdehyde dehydrogenase and a systematic review of the literature of reported ALDH5A1 mutations. J Perinat Med. 2016;44:441-51.

12. Jiang SZ, Shu JB, Zhang YQ, Fan WX, Meng YT, Song L. Analysis of ALDH5A1 gene mutation in a Chinese Han family with succinic semialdehyde dehydrogenase deficiency. Zhonghua Yi Xue Yi Chuan Xue Za Zhi. 2013:30:389-93.

13. Jakobs C, Bojasch M, Monch E, Rating D, Siemes H, Hanefeld F. Urinary excretion of gamma-hydroxybutyric acid in a patient with neurological abnormalities. The probability of a new inborn error of metabolism. Clin Chim Acta. 1981;111:169-78

14. Attri SV, Singhi P, Wiwattanadittakul N, Goswami JN, Sankhyan N, Salomons GS, et al. Incidence and geographic distribution of succinic Semialdehyde dehydrogenase (SSADH) deficiency. JIMD Rep. 2017;34:111-5.

15. Bertini E, Gospe SM Jr. SSADH deficiency in an adult: venturing outside of the diagnostic box and inside of the registry. Neurology. 2015;85:842-3.

16. Pearl PL, Gibson KM, Cortez MA, Wu Y, Carter Snead O 3rd, Knerr I, et al. Succinic semialdehyde dehydrogenase deficiency: lessons from mice and men. J Inherit Metab Dis. 2009;32:343-52.

17. Gibson KM, Gupta M, Pearl PL, Tuchman M, Vezina LG, Snead OC 3rd, et al. Significant behavioral disturbances in succinic semialdehyde dehydrogenase (SSADH) deficiency (gamma-hydroxybutyric aciduria). Biol Psychiatry. 2003;54:763-8.

18. Pearl PL, Novotny EJ, Acosta MT, Jakobs C, Gibson KM. Succinic semialdehyde dehydrogenase deficiency in children and adults. Ann Neurol. 2003;54(Suppl 6):S73-80

\section{Ready to submit your research? Choose BMC and benefit from:}

- fast, convenient online submission

- thorough peer review by experienced researchers in your field

- rapid publication on acceptance

- support for research data, including large and complex data types

- gold Open Access which fosters wider collaboration and increased citations

- maximum visibility for your research: over $100 \mathrm{M}$ website views per year

At BMC, research is always in progress.

Learn more biomedcentral.com/submissions 